\title{
EXTREME POINTS OF SUBCLASSES OF CLOSE-TO-CONVEX FUNCTIONS
}

\author{
H. SILVERMAN ${ }^{1}$ AND D. N. TELAGE
}

\begin{abstract}
We determine coefficient bounds, distortion and covering theorems, and the extreme points for various subclasses of close-to-convex functions. All results are sharp.
\end{abstract}

1. Introduction. Let $\mathcal{S}$ denote the class of functions of the form $f(z)=z+$ $\sum_{n=2}^{\infty} a_{n} z^{n}$ that are analytic and univalent in the unit disk $\mathcal{Q}$. A normalized function $f$ is said to be close-to-convex if there exists a function

$$
g(z)=b_{1} z+\cdots \quad\left(\operatorname{Re} b_{1}>0\right)
$$

starlike with respect to the origin for which

$$
\operatorname{Re}\left\{z f^{\prime} / g\right\}>0 \quad(z \in \mathcal{Q}) .
$$

It is well known [3] that the close-to-convex functions, denoted by $\mathcal{C}$, are contained in $\delta$.

In this paper we investigate distortion properties, coefficient bounds, and the extreme points of several subclasses of $\mathcal{C}$. A function $f$ is said to be in $C_{1}$ if there exists a convex function $g$ of the form (1) such that (2) is satisfied. If there exists such a $g$ satisfying

$$
\operatorname{Re}\left\{\left[z f^{\prime}\right]^{\prime} / g^{\prime}\right\}>0 \quad(z \in \mathcal{Q})
$$

then $f$ is said to be in $C_{2}$. If

$$
\operatorname{Re}\left\{\left[z\left[z f^{\prime}\right]^{\prime}\right]^{\prime} /\left[z g^{\prime}\right]^{\prime}\right\}>0 \quad(z \in \mathcal{Q}),
$$

then $f$ is said to be in $\mathcal{C}_{3}$. In relating these classes to one another, we will rely on the following lemma due to Sakaguchi [4].

Lemma A. Let $F(z)=z+\cdots$ be analytic and $G(z)=b_{1} z+\cdots$ be analytic and starlike in $\mathcal{Q}$ with $\operatorname{Re} b_{1}>0$. If $\operatorname{Re} F^{\prime} / G^{\prime}>0(z \in \mathcal{Q})$, then $\operatorname{Re} F / G>0(z \in \mathcal{U})$.

Since $g$ convex implies $z g^{\prime}$ is starlike, an application of Lemma A shows that $C_{3} \subset C_{2}$. Reapplying Lemma $A$ we see that $C_{2} \subset C_{1}$. Further $C_{1} \subset \mathcal{C}$

Received by the editors January 23, 1978 and, in revised form, March 20, 1978.

AMS (MOS) subject classifications (1970). Primary 30A32, 30A40; Secondary 30A34.

Key words and phrases. Univalent, starlike, convex, close-to-convex, extreme point.

${ }^{1}$ The research of the first author was supported in part by a College of Charleston summer research grant, and was completed while visiting at the University of Kentucky. 
because convex functions are starlike. We thus have the inclusion relations $\varrho_{3} \subset \varrho_{2} \subset \varrho_{1} \subset e_{\text {. }}$

Geometrically, a function $f$ is in the family $C_{2}$ if $z f^{\prime}$ maps each circle $z=r e^{i \theta}(r<1)$ onto a simple closed curve whose unit tangent vector never drops back on itself more than $\pi$ radians as $\theta$ increases. That is, $f \in \mathcal{C}_{2}$ if and only if $z f^{\prime} \in \mathcal{C}$. The family $\mathcal{C}_{1}$, while a proper subclass of the close-to-convex functions, is not contained in the family of starlike functions. In fact, there exist functions in $\mathcal{C}_{2}$ that are not starlike. For example, the function

$$
h(z)=\frac{1-i}{2} \frac{z}{1-z}-\frac{1+i}{2} \log (1-z)
$$

is shown in the next section to be in $\bigodot_{2}$. However for $\varepsilon$ sufficiently small, $\operatorname{Re}\left(z h^{\prime}(z) / h(z)\right)<0$ when $z=e^{i \theta},-\varepsilon<\theta<0$.

2. Extreme points of $\mathcal{C}_{1}$ and $\mathcal{C}_{2}$. For a compact family $\mathscr{F}$, we denote the closed convex hull of $\mathcal{F}$ by cl co $\mathscr{F}$ and the extreme points of cl co $\mathscr{F}$ by $\varepsilon(\mathrm{cl}$ co $\mathscr{F})$.

TheOREM 1. Let $X$ be the torus $\{(x, y)|x|=|y|=1\}, P$ be the set of probability measures on $X$,

$$
k(z, x, y)=(1+x) \frac{z}{1-y z}+x \bar{y} \log (1-y z),
$$

where $z \in \mathcal{Q}$ and $|x|=|y|=1$, and let $\mathcal{F}$ be the set of functions $f_{\mu}$ defined by

$$
f_{\mu}(z)=\int_{X} k(z, x, y) d \mu(x, y), \quad \mu \in \mathscr{P} .
$$

Then

$$
\operatorname{clco} \mathcal{C}_{1}=\mathscr{F}
$$

and

$$
\mathcal{E}\left(\operatorname{cl} \operatorname{co} \mathcal{C}_{1}\right)=\{k(z, x, y) \mid x \neq-1\} .
$$

Proof. Our proof will follow along the lines of the proof for $\mathcal{E}(\mathrm{cl} \operatorname{co} \mathcal{C})$, found in [1]. We first show that $\operatorname{cl} \operatorname{co} \mathcal{C}_{1} \subset \mathcal{F}$. If $f \in \mathcal{C}_{1}$, then $p(z)=$ $z f^{\prime}(z) / g(z)$ has positive real part in $\mathcal{Q}$ for some convex function $g$. By Herglotz' theorem, we can express $p(z)$ as

$$
p(z)=\int_{\Gamma} \frac{p(0) u+\overline{p(0)} z}{u-z} d \alpha(u)
$$

for some $\alpha$ a probability measure on the unit circle $\Gamma$. In [1] it is shown that we can express $g(z)$ as

$$
g(z)=\int_{\Gamma} \frac{g^{\prime}(0) z}{1-v z} d \beta(v),
$$

where $\beta$ is also a probability measure on $\Gamma$. Since $g^{\prime}(0) p(0)=1$, we use (3), (4) 
and Fubini's theorem to obtain

$$
\begin{aligned}
f^{\prime}(z) & =\int_{\Gamma} \frac{u+g^{\prime}(0) \overline{p(0)} z}{u-z} d \alpha(u) \cdot \int_{\Gamma} \frac{1}{1-v z} d \beta(v) \\
& =\int_{X} \frac{1+\varepsilon \bar{u} z}{(1-\bar{u} z)(1-v z)} d \alpha(u) d \beta(v),
\end{aligned}
$$

where $\varepsilon=\overline{p(0)} g^{\prime}(0)$ satisfies $|\varepsilon|=1$. To show that $f \in \mathcal{F}$ it is sufficient to show that the kernel functions in (5) belong to $\mathcal{F}^{\prime}$, the set of derivatives of functions belonging to $\mathscr{F}$. By a theorem in [1], given $u$ and $v$ there is a probability measure $\gamma$ on $\Gamma$ such that

$$
\frac{1+\varepsilon \bar{u} z}{(1-\bar{u} z)(1-v z)}=\int_{\Gamma} \frac{1+\varepsilon \bar{u} z}{(1-w z)^{2}} d \gamma(w) .
$$

Thus we need only show for arbitrary $w,|w|=1$, that we can find $x, y$, $|x|=|y|=1$, such that

$$
\frac{d}{d z} k(z, x, y)=\frac{1+x y z}{(1-y z)^{2}}=\frac{1+\varepsilon \bar{u} z}{(1-w z)^{2}} .
$$

Choosing the unit point mass $k(z, x, y)=k(z, \varepsilon \bar{w} \bar{u}, w)$, we see that cl co $\bigodot_{1}$ $\subset \mathcal{F}$.

To show that $\mathscr{F} \subset$ cl co $\bigodot_{1}$, we need only show that $\{k(z, x, y)\} \subset \bigodot_{1}$ for $|x|=|y|=1$. Choose a complex number $\delta=\delta(x)$ so that $\operatorname{Re}\{\delta(1+x y z) /(1$ $-y z)\}>0$. Since $g(z)=z / \delta(1-y z)$ is convex, we have

$$
\operatorname{Re} \frac{z d k(z, x, y) / d z}{g(z)}=\operatorname{Re} \frac{\delta(1+x y z)}{1-y z}>0,
$$

which shows that $\{k(z, x, y)\} \subset \bigodot_{1}$.

Thus the only possible extreme points for $\mathcal{C}_{1}$ are $\{k(z, x, y)\}$. Taking $g=f$ in the definition of $C_{1}$ and noting that convex functions are starlike, we see that $C_{1}$ contains the convex functions. Since $k(z,-1, y)=-\bar{y} \log (1-y z)$ is convex but is not an extreme point of the closed convex hull of convex functions, it cannot be an extreme point of the larger set cl co $\bigodot_{1}$.

Excluding $x_{0}=-1$ from consideration, it suffices to show that for each $x_{0}$, $y_{0},\left|x_{0}\right|=\left|y_{0}\right|=1$,

$$
k\left(z, x_{0}, y_{0}\right)=\int_{X} k(z, x, y) d \mu(x, y)
$$

is possible only if $\mu$ is a unit point mass at $\left(x_{0}, y_{0}\right)$. Differentiating both sides of (6) with respect to $z$, we obtain

$$
\frac{1+x_{0} y_{0} z}{\left(1-y_{0} z\right)^{2}}=\int_{X} \frac{1+x y z}{(1-y z)^{2}} d \mu(x, y) .
$$


Setting $z=\bar{y}_{0} r$ and letting $r \rightarrow 1^{-}$, we have

$$
1+x_{0}=\lim _{r \rightarrow 1^{-}} \int_{X}\left(\frac{1-r}{1-y \bar{y}_{0} r}\right)^{2}\left(1+x y \bar{y}_{0} r\right) d \mu(x, y) .
$$

Since the integrand in (7) is bounded by 2 , we may apply the Lebesgue bounded convergence theorem to obtain

$$
1+x_{0}=\int_{\Gamma \times\left\{y_{0}\right\}}(1+x) d \mu(x, y) .
$$

Setting $\Gamma_{0}=\Gamma \times\left\{y_{0}\right\}$ and $a=\mu\left(\Gamma_{0}\right)$, we have $0 \leqslant a \leqslant 1$ and

$$
1+x_{0}=a+\int_{\Gamma_{0}} x d \mu(x, y) \text {. }
$$

Since $\left|x_{0}+(1-a)\right|=\left|\int_{\Gamma_{0}} x d \mu(x, y)\right| \leqslant a$ and $\left|x_{0}+(1-a)\right| \geqslant\left|x_{0}\right|-(1-$ $a)=a$, we must have $x_{0}=-1$ or $a=1$. Since $x_{0} \neq-1$, it follows that $a=1$. From (8) we have $x_{0}=\int_{\Gamma_{0}} x d \mu(x, y)$, which can hold only if $\mu$ is a unit point mass at $\left(x_{0}, y_{0}\right)$.

COROLlaRY 1. If $f(z)=z+\sum_{n=2}^{\infty} a_{n} z^{n} \in \mathcal{C}_{1}$, then $\left|a_{n}\right| \leqslant 2-1 / n$, with equality for $k(z, 1,-1)$.

Proof. We need only consider $f \in \mathcal{C}_{1}$ of the form $k(z, x, y)$. It is easy to see that the modulus of the coefficients of $k$ are maximized when $x=1$ and $y=-1$.

Similarly we have

Corollary 2. If $f \in \mathcal{C}_{1}$, then

$$
\frac{2 r}{1+r}-\log (1+r) \leqslant|f(z)| \leqslant \frac{2 r}{1-r}+\log (1-r) \quad(|z| \leqslant r)
$$

and

$$
\frac{1-r}{(1+r)^{2}} \leqslant\left|f^{\prime}(z)\right| \leqslant \frac{1+r}{(1-r)^{2}} \quad(|z| \leqslant r),
$$

with equality for $k(z, 1,1)$ at $z= \pm r$.

We can use similar arguments to determine the extreme points of $C_{2}$. But we will use known results for $\mathcal{C}$ to give a quicker proof.

Theorem 2. Let $X$ be the torus $\{(x, y)|| x|=| y \mid=1\}$, $\mathscr{P}$ be the set of probability measures on $X$,

$$
h(z, x, y)=\frac{1-x \bar{y}}{2} \frac{z}{1-y z}-\frac{1+x \bar{y}}{2} \bar{y} \log (1-y z)
$$

for $|x|=|y|=1$, and let $\mathscr{F}$ be the set of functions $f_{\mu}$ defined by

$$
f_{\mu}(z)=\int_{X} h(z, x, y) d \mu(x, y) \quad(\mu \in \mathscr{P}) .
$$


Then

$$
\operatorname{clco} \bigodot_{2}=\mathscr{F}
$$

and

$$
\mathcal{E}\left(\operatorname{cl} \operatorname{co} \bigodot_{2}\right)=\{h(z, x, y) \mid x \neq y\} \text {. }
$$

Proof. Observe that $f \in \mathcal{C}_{2}$ if and only if $z f^{\prime} \in \mathcal{C}$. Thus the operator $L$ defined by $L(f)=\int_{0}^{z} f(\zeta) / \zeta d \zeta$ is a linear homeomorphism on the space of analytic functions with $L(\mathcal{C})=\bigodot_{2}$. Since

$$
h(z, x, y)=\int_{0}^{z} \frac{1-(x+y) \zeta / 2}{(1-y \zeta)^{2}} d \zeta
$$

the result follows from the results for $C$ proved in [1].

Corollary. If $f(z)=z+\sum_{n=2}^{\infty} a_{n} z^{n} \in \mathcal{C}_{2}$, then $\left|a_{n}\right| \leqslant 1$ and

$$
\begin{aligned}
& \frac{r}{1+r} \leqslant|f(z)| \leqslant \frac{r}{1-r} \quad(|z| \leqslant r), \\
& \frac{1}{(1+r)^{2}} \leqslant\left|f^{\prime}(z)\right| \leqslant \frac{1}{(1-r)^{2}} \quad(|z| \leqslant r) .
\end{aligned}
$$

Equality in all cases is obtained for $f(z)=z /(1-z)$.

REMARKs. The extreme points of both $\mathcal{C}_{1}$ and $\mathcal{C}_{2}$ are linear combinations of the extreme points of the convex functions and the functions convex of order $\frac{1}{2}$. See [2]. Setting $x=-y$, we see that the extreme points of convex functions are contained in those for $C_{2}$.

3. The class $C_{3}$. The standard techniques cannot be applied to determine the extreme points of $\mathrm{clco} \mathrm{C}_{3}$ because of the presence of an additional parameter in the numerator. Nevertheless we still have sharp coefficient bounds and distortion theorems for the class $e_{3}$.

TheOREM 3. If $f(z)=z+\sum_{n=2}^{\infty} a_{n} z^{n} \in \bigodot_{3}$, then $\left|a_{n}\right| \leqslant 2 / 3+1 / 3 n^{2}$. This result is sharp, with equality for

$$
f(z)=\frac{2}{3} \frac{z}{1-z}-\frac{1}{3} \int_{0}^{z} \frac{\log (1-\zeta)}{\zeta} d \zeta .
$$

Proof. If $f \in \bigodot_{3}$, then there exists a convex function $g(z)=\sum_{n=1}^{\infty} b_{n} z^{n}$ and a function of positive real part $p(z)=\sum_{n=0}^{\infty} c_{n} z^{n}$ with $\left|b_{1}\right|=\left|c_{0}\right|=1$ such that $\left[z\left[z f^{\prime}\right]^{\prime}\right]^{\prime}=\left[z g^{\prime}\right]^{\prime} p$. Then

$$
\left[z\left[z f^{\prime}\right]^{\prime}\right]^{\prime}=\sum_{n=1}^{\infty} n^{3} a_{n} z^{n-1}=\left(\sum_{n=1}^{\infty} n^{2} b_{n} z^{n-1}\right)\left(\sum_{n=0}^{\infty} c_{n} z^{n}\right) .
$$

Equating coefficients, we have $n^{3} a_{n}=\sum_{k=1}^{n} k^{2} b_{k} c_{n-k}$. It is well known that $\left|b_{n}\right| \leqslant 1$ and $\left|c_{n}\right| \leqslant 2$ for $n \geqslant 1$. Hence

$$
n^{3}\left|a_{n}\right| \leqslant 2 \sum_{k=1}^{n-1} k^{2}+n^{2}=\frac{n(n-1)(2 n-1)}{3}+n^{2}
$$


which simplifies to $\left|a_{n}\right| \leqslant \frac{2}{3}+1 / 3 n^{2}$. To show that the extremal function is in $\bigodot_{3}$, we take $g(z)=z /(1-z)$.

THEOREM 4. If $f \in \bigodot_{3}$, then

$$
\begin{aligned}
& \frac{2}{3} \frac{r}{1+r}+\frac{1}{3} \int_{0}^{r} \frac{\log (1+t)}{t} d t \\
& \quad \leqslant|f(z)| \leqslant \frac{2}{3} \frac{r}{1-r}-\frac{1}{3} \int_{0}^{r} \frac{\log (1-t)}{t} d t \quad(|z| \leqslant r), \\
& \frac{2}{3} \frac{1}{(1+r)^{2}}+\frac{1}{3} \frac{\log (1+r)}{r} \\
& \leqslant\left|f^{\prime}(z)\right| \leqslant \frac{2}{3} \frac{1}{(1-r)^{2}}-\frac{1}{3} \frac{\log (1-r)}{r} \quad(0<|z| \leqslant r) .
\end{aligned}
$$

Equality holds in all cases for the extremal function of Theorem 3.

Proof. Setting $h=z f^{\prime}$, we may write $\left[z h^{\prime}\right]^{\prime}=p g^{\prime}$, where $p(z)$ is a function of positive real part, $g(z)$ is a starlike function, and $|p(0)|=\left|g^{\prime}(0)\right|=1$. It is well known that $(1-r) /(1+r)<|p(z)|<(1+r) /(1-r)$ and $(1-r) /(1$ $+r)^{3}<\left|g^{\prime}(z)\right|<(1+r) /(1-r)^{3}$ for $|z|<r$. Hence

$$
\frac{(1-r)^{2}}{(1+r)^{4}}<\left|\left[z h^{\prime}(z)\right]^{\prime}\right| \leqslant \frac{(1+r)^{2}}{(1-r)^{4}} \quad(|z| \leqslant r) .
$$

Integrating along the straight line segment from the origin to $z=r e^{i \theta}$ in the right inequality of (9) we obtain

$$
\left|z h^{\prime}(z)\right| \leqslant \int_{0}^{r} \frac{(1+t)^{2}}{(1-t)^{4}} d t=\frac{3 r+r^{3}}{3(1-r)^{3}} \quad(|z|=r) .
$$

Now for every $r$ choose $z_{0},\left|z_{0}\right|=r$, such that $\left|h^{\prime}\left(z_{0}\right)\right|=\min _{|z|=r}\left|h^{\prime}(z)\right|$. If $L\left(z_{0}\right)$ is the pre-image of the segment $\left\{0, z_{0} h^{\prime}\left(z_{0}\right)\right\}$, then

$$
\begin{aligned}
\left|z h^{\prime}(z)\right| & \geqslant\left|z_{0} h^{\prime}\left(z_{0}\right)\right|=\int_{L\left(z_{0}\right)}\left|\left(z h^{\prime}(z)\right)^{\prime}\right||d z| \\
& \geqslant \int_{0}^{r} \frac{(1-t)^{2}}{(1+t)^{4}} d t=\frac{3 r+r^{3}}{3(1+r)^{3}} .
\end{aligned}
$$

In view of (10) and (11),

$$
\frac{3+r^{2}}{3(1+r)^{3}} \leqslant\left|\left[z f^{\prime}(z)\right]^{\prime}\right| \leqslant \frac{3+r^{2}}{3(1-r)^{3}} \quad(|z|=r) .
$$

Using again the method that gave us (10) and (11), we obtain

$$
\frac{2}{3} \frac{r}{(1+r)^{2}}+\frac{1}{3} \log (1+r) \leqslant\left|z f^{\prime}(z)\right| \leqslant \frac{2}{3} \frac{r}{(1-r)^{2}}-\frac{1}{3} \log (1-r) \text {. }
$$


One more application yields

$$
\begin{aligned}
\frac{2}{3} \frac{r}{1+r} & +\frac{1}{3} \int_{0}^{r} \frac{\log (1+t)}{t} d t \\
& \leqslant|f(z)| \leqslant \frac{2}{3} \frac{r}{1-r}-\frac{1}{3} \int_{0}^{r} \frac{\log (1-t)}{t} d t .
\end{aligned}
$$

The coefficient bounds give some indication as to the degree of containment of $e_{3} \subset e_{2} \subset C_{1}$. Another measure is the following covering theorem.

THEOREM 5. The disk $\mathscr{Q}$ is mapped onto a domain that contains the disk $|w|<1-\log 2 \approx 0.31$ by any $f \in \mathcal{C}_{1}$, onto a domain that contains the disk $|w|<0.50$ by any $f \in \mathcal{C}_{2}$, and onto a domain that contains the disk $|w|<\left(\pi^{2}\right.$ $+12) / 36 \approx 0.61$ by any $f \in C_{3}$.

Proof. Let $r \rightarrow 1^{-}$in the lower bound of the distortion results for $f$ in the three classes.

\section{REFERENCES}

1. L. Brickman, T. H. MacGregor and D. R. Wilken, Convex hulls of some classical families of univalent functions, Trans. Amer. Math. Soc. 156 (1971), 91-107.

2. L. Brickman, D. J. Hallenbeck, T. H. MacGregor and D. R. Wilken, Convex hulls and extreme points of families of starlike and convex mappings, Trans. Amer. Math. Soc. 185 (1973), 413-428.

3. W. Kaplan, Close-to-convex schlicht functions, Michigan Math. J. 1 (1952), 169-185.

4. K. Sakaguchi, On a certain univalent mapping, J. Math. Soc. Japan 11 (1959), 72-75.

Department of Mathematics, College of Charleston, Charleston, South Carolina 29401

Department of Mathematics, University of KentuCKy, LeXINGTon, KentuCKy 40506 